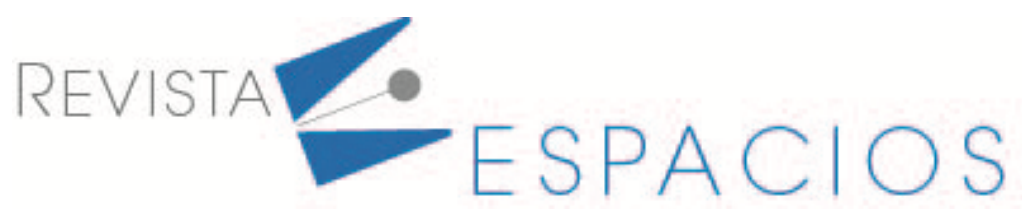

\title{
Competitividad del sector floricultor mediante la estrategia liderazgo en costos
}

\section{Competitiveness of the floricultural sector through the cost leadership strategy}

\author{
CALDERÓN, Luis C. ${ }^{1}$ \\ GUERRA, Vinicio R. ${ }^{2}$ \\ GALLEGOS, Mónica C. ${ }^{3}$ \\ BELTRÁN, Ligia I. 4
}

\begin{abstract}
Resumen
La presente investigación tuvo como propósito describir las características del liderazgo en costos en la competitividad del sector floricultor del Ecuador. Mediante una investigación mixta de alcance descriptivo se aplicó un instrumento dirigido a empresas florícolas del Cantón Pedro Moncayo. Los resultados muestran que tanto lascaracterísticas del liderazgo en costos, el bajo crecimiento del sector, el desconocimiento de la estructura de costos, el margen de ganancia y precios, han sido determinantes en la limitada competitividad del sector floricultor en el mercado internacional. Por tanto, los precios no han contribuido a generar una rentabilidad satisfactoria para los floricultores.

Palabras clave: floriculture, competitiveness, cost leadership, prices
\end{abstract}

\section{Abstract}

The purpose of this research was to describe the characteristics of cost leadership in the competitiveness of the Ecuadorian floriculture sector. Through a mixed investigation of descriptive scope an instrument was applied to flower companies of the Canton Pedro Moncayo. The results show that both the characteristics of cost leadership, the low growth of the sector, the ignorance of the cost structure, the margin of profit and prices, have been decisive in the limited competitiveness of the floriculture sector in the international market. Therefore, the prices have not contributed to generate a satisfactory profitability for the floriculturists.

Keywords: flower culture, competitiveness, cost leadership, prices

\footnotetext{
${ }^{1}$ Docente Investigador Facultad de Ciencias Administrativas y Económicas. Universidad Técnica del Norte, Ecuador. Magister en Dirección de Empresas. Iccalderon@utn.edu.ec

2 Docente Investigador Facultad de Ciencias Administrativas y Económicas. Universidad Técnica del Norte, Ecuador. Magister en Dirección de Empresas. evguerra@utn.edu.ec

${ }^{3}$ Docente Investigador Facultad de Ciencias Administrativas y Económicas. Universidad Técnica del Norte, Ecuador. Magister en Tecnologías para la Gestión.mgallegos@utn.edu.ec

${ }^{4}$ Docente Investigador Facultad de Ciencias Administrativas y Económicas. Universidad Técnica del Norte. Magister en Gestión Empresarial.

libeltran@utn.edu.ec
} 


\section{Introducción}

Desde los años 90 el sector florícola del Ecuador empieza a desarrollar un dinamismo en los cultivos de producción de flores para exportación y hoy en día es uno de los motores del comercio exterior que aporta significativamente a la economía ecuatoriana. Las flores del Ecuador son exportadas a diversos mercados internacionales como Estados Unidos, Rusia, Holanda, entre otros donde gozan del prestigio y reconocimiento de estos mercados internacionales, destacándose su excelente calidad y belleza, gracias al aprovechamiento de las condiciones climáticas que constituyen como una ventaja competitiva para el sector. Sin embargo, se requiere fortalecer sus estándares de calidad para ganar mayor competitividad en lo referente a MIPYMES. La presente investigación busca describir las características de las estrategias basadas en liderazgo en Costos que utilizan las florícolas ya posesionadas en el mercado que les ha permitido ser competitivas al ofrecer productos de menor costo en mercados nacionales e internacionales.

\section{Marco Teórico}

Contar con una estrategia es un aspecto fundamental para ganar competitividad en una empresa. Sin embargo, para (López, Corrales y Parra, 2018) la eficiencia en las organizaciones se limita a la disminución de costos e incremento de las utilidades.

(Lavielle, 2013), plantea que las empresas para ser competitivas, tienen la necesidad de ofrecer productos o servicios de calidad, al menor costo, que satisfagan las expectativas de los clientes y que garanticen ingresos sostenidos a la organización.

Sin embargo, no todo es satisfacción para los empresarios que toman decisiones, también hay dificultades que resolver; su papel va más allá de producir y generar beneficios; hay que garantizar según (Gómez, 2018) una metodología confiable para calcular los costos de los procesos, actividades y productos o servicios para luego diseñar un procedimiento que permita un mejoramiento continuo para alcanzar metas. Por ello (Porter, 2010a), considera importante trabajar en tres aristas: 1 . Liderazgo en costes, 2. Diferenciación y 3. Segmentación.

Para (Aulakh, Kotabe, \& Teegen, 2000), citado en (Fernández \& García-Pardo, 2008), manifiesta que a partir de las estrategias competitivas, existe un grado de adaptación o estandarización de las actividades de marketing en los mercados exteriores y la estrategia de expansión, esto es, el grado de diversificación o alcance geográfico de las exportaciones. Al respecto, (Porter, 2007) manifiesta que la ventaja en liderazgo en costos es uno de los dos tipos de ventaja competitiva que posee una empresa, es decir no se conseguirá un desempeño sobresaliente si el precio alto resultante no supera el costo de diferenciación. Está claro que las empresas tienen que cumplir con este requisito, de lo contrario tendrá que bajar los precios por una diferenciación no percibida por el cliente, por lo que la estrategia de liderazgo en costos se vendría abajo.

Para que esto no suceda (Jiménez Ramírez, 2006), propone que las fórmulas utilizadas por los gerentes para tornar competitivas sus empresas van desde la reducción de costos, despido de trabajadores, presión a la fuerza de ventas, hasta la implantación de nuevos modelos de administración y gestión empresarial. Según (Porter, 2010a) una empresa será un participante destacado de su sector industrial si logra alcanzar un liderazgo sostenible en costes (segmentación basada en costes) o en la diferenciación (segmentación basada en diferenciación). Ese fue el caso de las rosas del Ecuador que, según el Ministerio de Comercio Exterior, más de 20 empresas contribuyeron para que las flores andinas adornen este evento histórico del Vaticano para la Iglesia Católica. Sin embargo, según el (GAD-cantón-Pedro-Moncayo, 2015), a pesar de tener un valor agregado significativo, su estructura corresponde a una economía primaria que mantiene un peso relativo del $64 \%$ del total; caracterizado por la venta de productos agropecuarios sin valor agregado, como materias primas, flores o alimentos sin mayor procesamiento. 
Según el (BCE, Banco_Central_del_Ecuador, 2019), hasta el año 2015 la situación de las empresas florícolas tenía un crecimiento promedio anual del 9,42\%, con variaciones negativas en el año $2015-10,71 \%$ y $-2,13$ en el año 2016; sin embargo, en el año 2017se observa un crecimiento del 9,84\% y un decrecimiento significativo $-3,35 \%$ en el año 2018 recuperación del -0,68\% en el año 2017. Cabe indicar que las flores de Ecuador se encuentran entre los principales productos agrícolas de exportación, y son la tercera actividad agrícola que más genera divisas para el país, después del banano y el camarón.

Un factor que contribuye a la calidad y productividad de las rosas en Ecuador son las condiciones climáticas que posee Ecuador, como la perpendicularidad de los rayos solares y luz natural todo el día, lo convierten en un país ideal para producir flores, mismas que se encuentran acreditadas en el exterior por su exclusividad, su larga vida en florero, tallos largos, grandes botones, calidad y belleza. Según (CIM, EXPOFLORES, 2015b), la importancia del sector floricultor en el Ecuador, radica en la contribución al fomento de empleo, generación de ingresos para el Estado vía impuestos y apoyo a la competitividad del sector floricultor en el mundo. Pero la situación de este sector no siempre fue así, ya que los empresarios tuvieron que sortear muchas dificultades para financiar sus operaciones, el talento humano con limitadas capacidades, know how en la gerencia y búsqueda de mercados.

Tal como describe Andrade, citado en (Alvarado, 2002), que evalúa la competitividad del sector floricultor para el período 1996 - 1998, y concluye que uno de los factores de mayor importancia para la consecución de una ventaja competitiva es el uso intensivo de la mano de obra calificada, cuya realidad para esta época era todo lo contrario, los floricultores utilizaban mano de obra casi en su totalidad, no calificada, no capacitada, ni evaluada. En la actualidad las cosas han cambiado, el talento humano es capacitado y evaluado, el sector exige una planificación y ejecución minuciosa. Según (CIM, CLÚSTER FLOR-EXPOFLORES, 2017), el sector floricultor ofrece

36.400 plazas de trabajo, que permite la producción de las flores de exportación: mismas que se encuentran entre los principales productos agrícolas de exportación, y son la tercera actividad agrícola que más genera divisas para el país, después del banano y el camarón, (CIM, EXPOFLORES, 2015a).

Por ello es importante dar respuesta al objetivo de la investigación, cuyo propósito es describir las características del liderazgo en costos en la competitividad del sector floricultor del cantón Pedro Moncayo.

\subsection{La competitividad Empresarial}

En el contexto internacional y sobre todo el proceso de mundialización exige a las organizaciones ser eficientes y eficaces en el manejo de los recursos financieros, humanos, naturales, tecnológicos, para poder enfrentar (...) el mercado nacional e internacional" (Labarca, 2007, p. 159). Para abordar el estudio de la competitividad es necesario remontarse a Adam Smith, David Ricardo, John Stuart Mill y Michael Porter. El primero planteó la teoría de las ventajas absolutas, la cual postula para que un país pueda exportar un bien es necesario que tenga ventaja absoluta en la producción de este bien.

Para Adam Smith, "la ventaja absoluta estaba basada en la especialización para minimizar costos absolutos (maximización del beneficio); era la vía para que un país obtuviera mayores ganancias y el comercio se convertía en el generador de crecimiento en la producción mundial", citado en (Lombana \& Rozas Gutiérrez, 2009, p. 4). A pesar de ser un clásico económico (Smith, 1987), analiza la importancia de la división del trabajo, cuyo progreso depende de la aptitud y destreza de la gente y que su origen no está en la sabiduría humana como invento; sino que es consecuencia de una necesidad gradual de una cierta propensión a la naturaleza humana, para cambiar y negociar la diversidad de cosas y productos. Para sustentar estos hallazgos e incorporarlos al tratamiento de la competitividad, se consideró el apartado que manifiesta que "La preocupación de los economistas por la prosperidad de las naciones ha llevado al desarrollo de diversas teorías que tratan de explicar por qué algunos países son más competitivos que otros" (Buendía Rice, 2013, p. 55). Según (Cabrera Martínez, López López, \& Ramírez Méndez, 2011), la factibilidad de que una empresa alcance y mantenga sus niveles de competitividad se 
concentra en las competencias distintivas o ventajas competitivas que desarrolle internamente y en los condicionamientos externos que le brindan tanto la industria o sector al que pertenece, como la región-país en la que se encuentra ubicada.

\subsection{Liderazgo en costos}

"El liderazgo en costos consiste en que la organización se propone convertirse en el fabricante de coste bajo de su industria, (...) con todo, no se puede prescindir de los criterios de diferenciación" (Porter, 2010a, p. 41). En "las pequeñas y medianas empresas tienen serias dificultades en la aplicación de los sistemas modernos de costeo como fuentes de ventaja competitiva" (González \& Pérez, 2015, p. 90). El liderazgo en costos es la estrategia genérica de Michael Porter, que es posible cuantificarla, cuyos resultados tienen que ser claros; sin embargo, el reto es bastante alto, el objetivo es convertirse en la industria o empresa que menos costos genera al producir un mismo producto. Al respecto, (Alvarez Pinto, 2007, p. 91), manifiesta que con la estrategia de liderazgo en costos "la organización se concentra en alcanzar los costos más bajos de producción y distribución lo cual le da la capacidad de establecer los precios en un nivel más bajo que sus competidores".

El propósito de trabajar en bajos costos es monopolizar el mercado meta al cual está dirigido el producto, o dicho de otro modo, el producto que va dirigido a una determinada población. Aunque no todo puede ser así, una empresa siempre busca operar con bajos costos para tener un mejor margen de ganancia. Al respecto (Cadena Lozano, 2011, p. 73), afirma que "Teniendo claras las dos teorías que explican el comportamiento de los ingresos y los costos, y asumiendo cualquier mercado de competencia imperfecta, el beneficio vendrá dado por la diferencia entre el total de ingreso y el costo total".

Además, sostiene que, para la fijación de precios, se considera tres disciplinas:1) la teoría económica que analiza precios, oferta y demanda;2) la teoría financiera fija el precio para proyectar ingresos y determinar márgenes de rentabilidad y 3) el marketing que propone planes de mercadeo para interactuar con las clientes de la empresa. Sin embargo, (Morillo \& Marisela, 2001a, p. 43), aconseja trabajar en la teoría del valor, cuyo aporte se fundamenta en "la percepción subjetiva y motivacional de los consumidores al comprar cierto artículo, (...), el valor se incrementa al satisfacer las necesidades mejor que la competencia, a un precio que no rebase las disponibilidades del consumidor".

Según (Porter, 2010b, p. 66), desde el punto de vista de la competencia, el valor es lo que la gente está dispuesta a pagar por lo que le ofrece. Otra propuesta que contribuye la reducción de costos desde la gestión es (Gitlow \& Gitlow, 1989), citado en (Valenzuela Jiménez, 2016, p. 64), quien afirma que "con la metodología Deming se mejoran los procesos del sistema productivo para lograr menos piezas defectuosas y más artículos de calidad con menor costo y mayor flexibilidad para los precios con los que se aumenta participación en los mercado.

Así mismo, (Mallo, Meljem, \& Jiménez, 2000, p. 41) señala que "la contabilidad de dirección o gestión, plantea la asignación eficiente de recursos, analizando las funciones de producción (...) la de costos y el comportamiento de los mercados. Su objetivo consiste en la maximización de beneficios. Los costos de producción son sin lugar a duda el insumo de la presente investigación, ya que marcan la pauta del éxito o fracaso de un negocio. Para entender la importancia de los costos (García-Colín, 1996, p. 10), manifiesta que "la contabilidad de costos industriales es un sistema de información empleado para predeterminar, registrar, acumular, distribuir, controlar, analizar, interpretar e informar de los costos de producción, distribución, administración y financiamiento".

Es por ello que existe un complejidad al asignar los costos a los productos o servicios, como afirma (Lafuente \& Paez, 2018), existe dificultad para identificar los generadores de costos, sin embargo persiste el criterio de los directivos en tomar decisiones basada en datos. 


\subsection{Margen de utilidad}

Autores como (Aguirre \& Romero, 2016), consideran que para el tratamiento de la utilidad es importante referirse a los ingresos o ventas, el costo de ventas, la utilidad bruta, los gastos generales, la utilidad operativa (EBITDA), ingresos no efectivos, utilidad operativa (EBIT) ingresos o gastos financieros, utilidad antes de impuestos, participación de utilidades e impuestos y la utilidad neta del ejercicio que es dividida a favor de los accionistas, para obtener la utilidad retenida. Según (Meigs, et al, 1999), citado en (Morillo \& Marisela, 2001b, p. 36), "la utilidad neta es el incremento del patrimonio resultante de una operación lucrativa de la empresa, esperado por quienes invierten en el capital social de una compañía para incrementar el valor de su inversión".

Si el margen de ganancia es mayor que el costo de capital se estaría hablando de un producto que podría tener una viabilidad económica financiera. Por lo tanto, los empresarios florícolas deben preocuparse por bajar sus costos de producción, con la finalidad de ser competitivos en costos y en precios. Respecto al precio, (Sapag Chain, Sapag Chain, \& Sapag, 2014), discute que la determinación del precio es un elemento de la estrategia comercial que junto con la demanda, define el nivel de ingresos de la organización.

En ese sentido el cálculo del precio debe tomar en cuenta las condiciones de comercialización (crédito), los porcentajes de cobro en efectivo y a plazo, el número y monto de las cuotas, la tasa de interés, los descuentos por pronto pago; de este conjunto de variables depende si una empresa es o no rentable.

\section{Metodología}

Con el objeto de identificar y caracterizar las principales estrategias de liderazgo en costos que están presentes en las florícolas y los beneficios que permiten mejorar su competitividad. Se desarrolla la presente investigación que se fundamenta en dos enfoques: 1)cualitativo dentro de la investigación acción, fusionando el componente florícola con las estrategias de liderazgo en costos que permite la entrevista como herramienta de recolección y mediante enfoque 2) cuantitativo de alcance descriptivo se utilizó la encuesta como instrumento para recoger la información sobre el proceso de caracterización de las empresas participantes, analizadas a través de procesos estadísticos.

En el análisis e interpretación de los datos se utilizó dos herramientas: la técnica focus group con los expertos en el área florícola y encuesta aplicada a los empresarios floricultores del cantón Pedro Moncayo. Para el focus group se seleccionó una muestra intencional de ocho profesionales expertos, quienes opinaron acerca de los indicadores. Se tomó en cuenta su experiencia y conocimiento en el sector florícola. Para la aplicación de los instrumentos se contó con la base de datos tomada de la Superintendencia de Compañías, se suministraron varios archivos en los que se presentaban listados con datos de empresas de diversos sectores y actividades económicas, sin embargo, se fortaleció esta información con los datos tomados del sitio Web de Expo Flores.

A partir de estos listados se construyó una base de datos con las empresas floricultoras. Así se identificaron 72 empresas en el sector de Cayambe. De ellas 37 respondieron el instrumento, esto corresponde al $51 \%$ de la población. El proceso de contactar a las empresas se hizo por varios medios: telefónicamente, a través de mail y mediante visitas. Dentro del modelo de análisis, se definieron las dimensiones y variables consideradas a partir de una revisión documental y el estado del arte para indagar la situación actual en cada una de las empresas.

Finalmente, se realizó en análisis e interpretación de datos donde se evidencia los resultados obtenidos en cada una de las preguntas del instrumento aplicado. 


\section{Resultados}

Para el análisis y discusión de resultados se tomó en consideración los siguientes factores:

\subsection{Tipo de empresas}

El 40\% de empresas del sector florícola del cantón Pedro Moncayo son microempresas, seguido de grandes, medianas y pequeñas empresas. Según (DEE, INEC, 2012), el sector micro empresarial representa el $89,6 \%$ del sector productivo a nivel nacional, seguido de pequeñas, medianas y grandes

empresas. Así mismo, en un estudio realizado por Audretsch y Thurik (2001), citado en (Flores, Lagarda, \& Urquidy, 2011), demuestra la importancia que la actividad empresarial tiene en términos del crecimiento económico de una región, reducción del nivel de desempleo, presencia de una estructura empresarial poco dinámica caracterizada por procesos de subsistencia, pero de valor social y económico como las microempresas.

\subsection{Tiempo de operación en el mercado floricultor}

Las empresas que operan entre 1 y 5 años representan el 34,3\%, las que vienen operando entre seis y quince años representan $31,4 \%$, mientras las empresas que vienen operando por más de diez y seis años representan el 34,3\%. Según (Bautista 2013), citado en (Fajardo Vaca, Fernández Ronquillo, Vásquez Fajardo, Toscano Ruiz, \& Fajardo Vaca, 2016, p. 5), las microempresas en Ecuador se han convertido en una fuerza estabilizadora y realizan una contribución importante al empleo, a la producción, al ingreso nacional y local.

\subsection{Características de las empresas}

Las empresas del sector floricultor del cantón Pedro Moncayo, se caracteriza en su mayoría por ser de tipo familiar, seguido de accionistas, socios y alianzas de empresas, cuya estructura en muchas ocasiones les ha traído inconvenientes como manifiesta (Ginebra, 2005, p. 17), "en las empresas familiares, existe quejas como cuando se hicieron cargo del negocio, para salvarlo tuvieron que mandar a la casa a una serie de parientes o amigos de parientes que parasitaban escandalosamente la marcha de la empresa". Así mismo, (Lozano Posso, 2000, p. 17), manifiesta que en las empresas familiares, "se producen disgustos entre padres e hijos, choque entre hermanos, sucesión inadecuada o inexistente, estados críticos de fluidez económica y limitación en las ganancias, desmotivación de miembros familiares o de empleados no familiares". Este puede ser uno de los factores determinantes que limitan el continuo crecimiento y desarrollo de este tipo de negocios.

\subsection{Forma de comercializar la flor}

El 62,9\% de empresarios manifiesta operar en mercado nacional e internacional, es decir su eje estratégico no es local, sino que dan a conocer sus productos por diversos medios digitales cuyo éxito está en exportar directamente la flor de Ecuador a Europa, Estos Unidos, Rusia y Países Bajos; mientras que los floricultores que no pueden exportar directamente lo hacen a través de intermediarios. Según (CIM, EXPOFLORES, 2018), la mayoría de las grandes empresas florícolas del Ecuador son exportadoras y se concentran en Quito o sus alrededores.

Según (Harari, Korovkin, Larrea, Martínez, \& Ortiz, 20014, p. 79), "la globalización ha incrementado los niveles de integración económica mundial, ha difundido nuevas tecnologías y ha propulsado un nuevo ciclo de crecimiento económico".

\subsection{Estado de crecimiento sector floricultor}

El 48,6\% de empresarios han manifestado su satisfacción por el crecimiento de sus negocios y el sector floricultor, sin embargo, el 48,6\% aseguran que el crecimiento del mercado se ha detenido, y el 2,9\% ha manifestado que el 
mercado florícola ha decrecido. Según (CIM, EXPOFLORES, 2015a), la demanda de flores a escala mundial registra un crecimiento del $1,7 \%$ con respecto al 2013.

Colombia, Kenia y Etiopía mantienen una tendencia creciente en sus exportaciones en los últimos años, mientras que Ecuador experimentó un decrecimiento en este último año. Sin embargo, en (CIM, EXPOFLORES, 2018), se determina que el sector florícola es una de las industrias más grandes del Ecuador. Actualmente el país cuenta con el $9 \%$ de la cuota de mercado mundial de flores, con esto logra consolidarse en el mercado nacional e internacional.

\subsection{Certificación de calidad y medio ambiente}

El 71,4\% de los empresarios del sector floricultor han manifestado que poseen una certificación de calidad, en ese sentido se determina y analiza un conjunto de factores de éxito que configuran una estrategia basada en la competitividad y calidad que les permite diferenciarse ya sea por precio o por los atributos del producto. (Olivares_Valdés, 2009).

En cuanto al cuidado del medio ambiente, (Santos Garcia et al., 2009, p. 83), en su investigación sobre el enfoque territorial de la calidad y su impacto en la competitividad afirma que los países en desarrollo solo pueden competir si cumplen con la calidad que exige el mercado y que la evidencia de ello es posible si se implanta una infraestructura de la calidad.

\subsection{Costos totales}

El $68,6 \%$ de los empresarios manifestaron no conocer los costos totales de sus empresas. No obstante, respecto al costo unitario de una rosa, el $74,3 \%$ de empresarios manifestaron conocer su costo, lo que significa que hay un alto interés que contribuye a la toma de decisiones. Referente a la competitividad el 65,7\% de empresarios del sector floricultor manifestaron que los costos de producción de las empresas son competitivos. Sin embargo, no solo hay que conocer únicamente los costos de producción de las empresas florícolas nacionales, sino también las del exterior, por compartir un mercado globalizado, pues apenas un 8,6\% manifestaron conocer dichos costos de producción de las empresas florícolas del exterior. Lo que significa un descuido en estar informados, sobre todo porque los exportadores e importadores, se esmeran en reducir costos, aprovechando cualquier estrategia disponible para lograr este propósito de competitividad entre empresas florícolas nacionales e internacionales. El 65,7\% de empresarios manifestaron que los costos de producción de las empresas florícolas en Ecuador no son competitivos con los costos de producción del mercado internacional. Según (Molina Paredes, 2003), para que las empresas logren la verdadera competitividad deben utilizar técnicas de gestión, tendientes a controlar y reducir costos, así como a mantener su posicionamiento en el mercado. Así mismo (Cuevas Villegas, 2002, p. 15), manifiesta que "la competencia hoy se extiende más allá de las fronteras de cada país, los costos y los precios se afectan por la llegada de competidores foráneos y por las fluctuaciones en las tasas de cambio". Una de las principales causas es la carga arancelaria que sufren este tipo de productos que encarecen su productividad.

Cuando se preguntó a los empresarios, sobre su nivel de producción y costos, el 45,7\% manifestaron que en su nivel óptimo de producción la empresa disminuye los costos por unidad. Así reconoce (Morillo \& Marisela, 2001b), las empresas no sólo requieren reducir costos por cuestiones relacionadas con las utilidades (enfoque interno), sino también por factores relacionados con la competencia (enfoque externo). En ese sentido (Porter, 2010b, p. 39) sostiene que "un negocio puede competir teniendo costos bajos y ofreciendo productos de calidad superior".

Finalmente, al relacionar costos de producción, calidad y durabilidad de las rosas, el 85,7\% de empresarios manifestaron que los costos nunca se verían afectados por dedicar recursos a la calidad y durabilidad de la flor. Más difícil es asumir el costo de la no calidad. Según (Márcio Carvalho, Marini Thome, \& Oliveira Leitao, 2014), 
manifiesta que las empresas que son incapaces de manejar problemas de calidad de manera satisfactoria encuentran poco lugar en el mercado.

\subsection{Margen de ganancia}

El 42,9\% de empresarios manifestaron no conocer el margen de ganancia que se grava al costo unitario para obtener la utilidad esperada. (Sapag Chain et al., 2014) considera que para calcular el margen sobre el precio o sobre los costos, se toma como referencia el costo de capital. En cambio (Cuevas Villegas, 2002, p. 15), manifiesta que "los márgenes de utilidad se establecen para obtener un rendimiento razonable sobre la inversión".

Al preguntar sobre el margen, costo y utilidad, el 57,1\% de empresarios manifestaron que el margen asignado a los costos de producción no les ha permitido obtener la utilidad esperada. Spencer (1963), citado en (Vergara Reina, Pozo Contrera, \& Ramos Pérez, 2014), dice que un fabricante que fija su precio según el método por encima del costo confronta dos problemas de decisión: 1) obtener una estimación del costo, y 2) elegir el margen o sobre marca apropiado.

En ese sentido respecto a los competidores asevera que todo negocio debe estar pendiente de las acciones de la competencia y sus precios siempre tendrán relación con los de la competencia, en el caso de productos similares y de fácil sustitución.

Finalmente, respecto al margen y utilidad, el 62,9\% de empresarios manifestaron que el precio de venta en el mercado internacional, no ha permitido obtener ganancias. Según (Romero \& Puente, 2018, p. 50), "los años de prosperidad para las flores ecuatorianas han quedado atrás". Desde el año 2015 el sector experimenta sobreoferta en el mercado internacional, pérdida de participación en mercados, devaluación de monedas de los principales competidores (Colombia y Kenia), y la caída de 10,71 \% del precio hasta noviembre de 2015. (BCE, Banco Central del Ecuador, 2019).

\section{Conclusiones}

Las características predominantes del liderazgo en costos que contribuyen a la competitividad del sector floricultor del cantón Pedro Moncayo-Ecuador, se describen a continuación:

La mayoría de empresas que vienen operando por más de diez y seis años son de tipo familiar, cuyo éxito de los empresarios es operar en el mercado nacional e internacional, exportar directamente la flor de Ecuador a Europa, Estos Unidos, Rusia y Países Bajos.

Los empresarios tienen un criterio dividido en cuanto al crecimiento, decrecimiento y estancamiento del mercado de flores en Ecuador y en el mundo.

Aunque parece contradictorio, muchos empresarios no conocen los costos totales, el costo unitario de una rosa, peor aún los costos de las empresas florícolas del mercado internacional.

Un grupo significativo de empresarios no conocen el margen de ganancia que se grava al costo unitario para obtener la utilidad esperada. En su mayoría están de acuerdo que los costos nunca se verían afectados por dedicar recursos a la calidad de la flor.

\section{Referencias bibliográficas}

Aguirre Gómez, L., \& Romero Morán, F. (2016). MANUAL DE FINANCIMAINETO PARA EMPRESAS.

Guayaquil-Ecuador. https://doi.org/978-9942-969-62-0 
Alvarado, S. (2002). Programa de Maestría en Relaciones Internacionales Mención Negociaciones Internacionales El caso del comercio exterior de laflor ecuatoriana como. Quito - Ecuador. Retrieved from http://repositorio.uasb.edu.ec/bitstream/10644/2668/1/T0154-MRI-Alvarado-El caso.pdf

Alvarez Pinto, F. D. (2007). Planificación Estrategica de Marketing. Perspectivas, 20(1), 67-104. Retrieved fromhttp://www.redalyc.org/pdf/4259/425942331006.pdf

Aulakh, P. S., Kotabe, M., \& Teegen, H. (2000). Export strategies and performance of firms from emerging economies : Evidence ... Retrieved from http://www.rcmewhu.com/upload/file/20150528/20150528105357_7021.pdf

Banco_Central_del_Ecuador. (2019). Información Estadís ca Mensual No . 1997 - Julio 2018. Retrieved fromhttps://contenido.bce.fin.ec/home1/estadisticas/bolmensual/IEMensual.jsp

Banco Central del Ecuador. (2019). Información Estadísca Mensual No . 2012 - Octubre 2019. Quito Ecuador. Retrieved fromhttps://contenido.bce.fin.ec/home1/estadisticas/bolmensual/IEMensual.jsp

Buendía Rice, E. A. (2013). El papel de la Ventaja Competitiva en el desarrollo económico de los países. Analisis Economico, XXVIII(69), 55-78. Retrieved from http://www.redalyc.org/pdf/413/41331033004.pdf

Cabrera Martínez, A. M., López López, P. A., \& Ramírez Méndez, C. (2011). La competitividad empresarial: un marco conceptual para su studio (Ediciones). Bogotá. Retrieved from https://mba.americaeconomia.com/sites/mba.americaeconomia.com/files/competitividad_peq_emp .pdf

Cadena Lozano, J. B. (2011). La teoría económica y financiera del precio: dos enfoques complementarios, 9(15), 59-80. Retrieved fromhttp://132.248.9.34/hevila/Criteriolibre/2011/vol9/no15/2.pdf

CLÚSTER FLOR-EXPOFLORES. (2017). Informe elaborado por el Área de Comunicación, Información y Marketing (CIM) de la Asociación de Productoresy Exportadores del Ecuador, Expoflores. Retrieved July 17, 2018, from http://flor.ebizor.com/sector-floricola-de-quito-lidera-mercado/

Cuevas Villegas, C. F. (2002). FIJACIÓN DE PRECIOS Costo Plus (Costo más margen) y Target Costing (Costeo Objetivo), 13-30.https://doi.org/0123-5923

EXPOFLORES. (2018). Top 10 de las empresas florícolas más grandes de Ecuador. Quito - Ecuador. Retrieved from http://flor.ebizor.com/top-10-de-las-empresas-floricolas-mas-grandes-de-ecuador/

EXPOFLORES, C. (2015a). Exportadores de flores. Quito - Ecuador. Retrieved from https://es.slideshare.net/florecuador/informe-principales-exportadores-de-flores-2015

EXPOFLORES, C. (2015b). Informe elaborado por el Área de Comunicación, Información y Marketing (CIM) de la Asociación de Productores y Exportadores del Ecuador, Expoflores. Retrieved November 22, 2019, from https://www.slideshare.net/florecuador/informe-principales-exportadores-de-flores2015

Fajardo Vaca, L., Fernández Ronquillo, M., Vásquez Fajardo, C., Toscano Ruiz, D., \& Fajardo Vaca, L. (2016). La Asociatividad Micro empresarial, comomecanismo productivo sostenible: Caso Cantón Milagro Ecuador TT - The microenterprise association, as a sustainable production mechanism: Canton Milagro case - Ecuador. Universidad, Ciencia y Tecnología, 20(78), 4-13. Retrieved from http://www.scielo.org.ve/scielo.php? script=sci_arttext\&pid=S131648212016000100001\&lang=pt\%0Ahttp://www.scielo.org.ve/pdf/uct/v20n78/art01.pdf 
Fernández, V., \& García-pardo, H. P. (2008). Factores determinantes del éxito exportador . El papel de la estrategia exportadora en las cooperativasagrarias, 1-27. Retrieved from http://www.redalyc.org/articulo.oa?id=17412307002

Flores, M. T., Lagarda, A. M., \& Urquidy, M. R. (2011). Aprendizaje en microempresas de Baja California Introducción La actividad empresarialcontribuye en la dinámica social y económica, 12, 95-116. Retrieved from http://www.scielo.org.mx/pdf/estfro/v12n23/v12n23a5.pdf

GAD-cantón-Pedro-Moncayo. (2015). Plan De Ordenamiento Y Desarrollo Turistico. Tabacundo. Retrieved fromhttp://www.pedromoncayo.gob.ec/documentos/ord2015/PDOT.pdf

García-Colín, J. (1996). Contabilidad de Costos. In M. de México (Ed.) (p. 270). México. Retrieved from https://ebookcentral.proquest.com/lib/utnortesp/reader.action?doclD=3196408\&query=COLIN

Ginebra, J. (2005). Las empresas familiares su dirección y continuidad (Sexta reim, p. 17). México.

Retrieved from https://books.google.es/books?

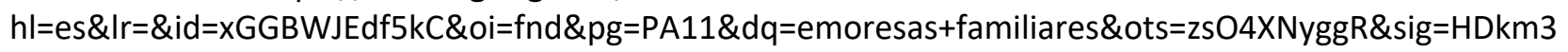
Q8yRVvis2vCLLjpSIYyIN4\#v=onepage \&q=emo familiares\&f=false

González, J., \& Pérez, Y. (2015). Análisis de los Sistemas de Costos como herramientas estratégicas de gestión en las Pequeñas Y Medianas Empresas(Pymes). Revista Publicando, 2(3), 90-113. Retrieved from https://rmlconsultores.com/revista/index.php/crv/article/view/31

Harari, R., Korovkin, T., Larrea, C., Martínez, L., \& Ortiz, P. (20014). EFECTOS SOCIALES DE LA GLOBALIZACIÓN Petróleo, banano y flores enEcuador. (C. C. de I. de los M. S. del Ecuador, Ed.). Ecuador-Quito. Retrieved from https://digitalrepository.unm.edu/cgi/viewcontent.cgi? referer=https://scholar.google.es/\&httpsredir=1\&article=1415\&context=abya_yala

INEC. (2012). Directorio de Empresas y Establecimientos Contenido Generalidades Resultados. Retrieved from http://www.ecuadorencifras.gob.ec/documentos/webinec/Estadisticas_Economicas/DirectorioEmpresas/140210 DirEmpresas final3.pdf

Jiménez Ramírez, M. H. (2006). Modelo De Competitividad Empresarial. Umbral Científico, (9), 115-125. Retrieved fromhttp://www.redalyc.org/pdf/304/30400913.pdf

Labarca, N. (2007). Consideraciones teóricas de la competitividad empresarial. OMNIA. Retrieved from http://www.redalyc.org/pdf/737/73713208.pdf

Lafuente Velazquez, M. R., \& Paez Von Schmeling, N. A. (2018). GESTIÓN DE COSTOS EN LAS PYMES- UN ANALISIS EN LAS INDUSTRIAS GRAFICASDEL PARAGUAY, 10, 84-105. https://doi.org/2105-8798

Lavielle, S. (2013). El registro y control de los costos de calidad. Un análisis alternativo , en la fabricación de un producto exportable, $V, 149-159$. Retrieved from https://revistas.uo.edu.cu/index.php/aeco/article/viewFile/556/531

Lombana, J., \& Rozas Gutiérrez, S. (2009). Marco analítico de la competitividad: Fundamentos para el estudio de la competitividad regional.

Pensamiento y Gestión, 26, 38. Retrieved from

http://rcientificas.uninorte.edu.co/index.php/pensamiento/article/viewFile/854/499

Lozano Posso, M. (2000). El protocolo en las Empresas de propiedad familiar. Estudios Gerenciales (Vol. 16). https://doi.org/10.1063/1.1704874

Mallo, C., Meljem, S., \& Jiménez, C. (2000). Contabilidad de costos y estratégias de gestión, 9, 768. Retrieved fromhttp://www.accid.org/revista/documents/La_gestion_del_circulante_como_1.pdf 
Márcio Carvalho, J., Marini Thome, K., \& Oliveira Leitao, F. (2014). LA GESTIÓN DE LA CALIDAD COMO RECURSO DE REDUCCIÓN DE COSTES DETRANSACCIÓN, 15(1), 174-199. Retrieved from http://web.b.ebscohost.com/ehost/pdfviewer/pdfviewer?vid=6\&sid=0f44e594-bf1d-429c-8cdac042f2c69bea\%40pdc-v-sessmgr03

Molina Paredes, O. R. (2003). Nuevas Técnicas de Control y Gestión de Costos en Búsqueda de la Competitividad. Actualidad Contablre FACES.https://doi.org/1316-8533

Morillo, \& Marisela. (2001a). Rentabilidad Financiera y Reducción de Costos, 4, 35-45. Retrieved from http://www.redalyc.org/articulo.oa?id=25700404

Morillo, \& Marisela. (2001b). Rentabilidad Financiera y Reducción de Costos. Actualidad Contable FACES, 4, 35-48. Retrieved fromhttp://www.redalyc.org/articulo.oa?id=25700404

OLIVARES_VALDÉS, O. (2009). Competitividad y calidad factores críticos que contribuyen al éxito de Empresas de Menor Tamaño en mercadosglobales de país emergente. Revista Chilena de Economía y Sociedad, 3(1), 29-51. Retrieved from

http://web.b.ebscohost.com/ehost/pdfviewer/pdfviewer?vid=0\&sid=688223d4-b51d-408f-be797733a770f107\%40sessionmgr120

Porter, M. (2007). Ventaja Competitiva Creación y sostenimiento de un desempeño superior. (J. E. C. Ing, Ed.) (The Free P). México.Porter, M. (2010a). Ventaja Competitiva. (PIRÁMIDE, Ed.) (2010th ed.). España.

Porter, M. (2010b). VENTAJA COMPETITIVA creación y sostenimiento de un rendimiento superior. (B. C. Eduardo, Ed.) (Ediciones). España.

Romero, B., \& Puente, P. (2018). Adiós a la época dorada del sector florícola. Retrieved from http://www.revistagestion.ec/sites/default/files/import/legacy_pdfs/262_004.pdf

Santos Garcia, E. M., Rodríguez Rosada, A., Ferenandes Clúa, M. de J., Dayamí González, A., Rosada, \& Fajardo García, R. (2009). El enfoque Territorial de la Calidad y su Impacto en la Competitividad, 36(1), 83-89. Retrieved from http://web.b.ebscohost.com/ehost/pdfviewer/pdfviewer? vid=0\&sid=cce03e8c-2c76-4337-ae6c-1739889f $270 f \% 40 p d c-v-s e s s m g r 02$

Sapag Chain, N., Sapag Chain, R., \& Sapag, J. M. (2014). Preparación y Evaluación de Proyectos. México. https://doi.org/978-956-278-206-7

Smith, A. (1987). Investigación sobre la naturaleza y causas de la riqueza de las naciones / Adam Smith. Retrieved from http://www.elrepublicano.com.ar/images/Descargas/LaRiquezadeLasNacionesAdamSmith.pdf

Valenzuela Jiménez, L. F. (2016). Los costos de la mala calidad como quinto elemento del costo: aproximación teórica en la gestión de lacompetitividad en medio de la convergencia contable. Rev.Fac.Cienc.Econ, XXIV(1), 63-84. https://doi.org/10.18359/rfce.1622

Vergara Reina, R., Pozo Contrera, A., \& Ramos Pérez, E. (2014). Metodología para el cálculo de precios de transferencia con margen de utilidad. Revista científica avances (Vol. 16). https://doi.org/1562_3297

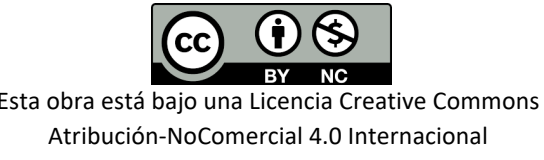

